\title{
High mRNA expression of GABA receptors in human sperm with oligoasthenoteratozoospermia and teratozoospermia and its association with sperm parameters and intracytoplasmic sperm injection outcomes
}

\author{
Paweena Kaewman ${ }^{1,2}$, Sutisa Nudmamud-Thanoi ${ }^{1,2}$, Patcharada Amatyakul ${ }^{3,4}$, Samur Thanoi ${ }^{1,2}$ \\ ${ }^{1}$ Department of Anatomy and ${ }^{2}$ Centre of Excellence in Medical Biotechnology, Faculty of Medical Science, Naresuan University, Phitsanulok; \\ ${ }^{3}$ Department of Obstetrics and Gynecology and ${ }^{4}$ Naresuan Infertility Centre, Faculty of Medicine, Naresuan University, Phitsanulok, Thailand
}

Objective: This study investigated the mRNA expression of gamma-aminobutyric acid (GABA) receptors in the sperm of oligoasthenoteratozoospermic (OAT) and teratozoospermic (TER) men compared to normozoospermic (NOR) men, as well as the relationships between GABA receptor expression and sperm parameters, fertilization rate, and embryo quality.

Methods: The mRNA expression of GABA A- $\alpha 1$ and GABA B-R2 receptors in sperm was examined using reverse transcription-polymerase chain reaction in three groups of patients: NOR $(n=32)$, OAT $(n=22)$, and TER $(n=45)$. The fertilization rate and embryo quality were assessed in 35 patients undergoing intracytoplasmic sperm injection (ICSI; 10 NOR, 10 OAT, and 15 TER men).

Results: OAT men had significantly higher mRNA expression of GABA A- $a 1$ and GABA B-R2 receptors in sperm than NOR men; however, the difference between TER and NOR men was not significant. High levels of these receptors were significantly correlated with low sperm concentration, motility, and morphology, as well as the rate of good-quality embryos (GQEs) at the cleavage stage after ICSI. Patients whose female partners had a $>50 \%$ GQE rate at the cleavage stage had significantly lower levels of GABA A-a1 receptor expression than those whose partners had a $\leq 50 \%$ GQE rate.

Conclusion: Our findings indicate that mRNA levels of GABA receptors in human sperm are correlated with poor sperm quality and associated with embryo development after ICSI treatment. The GABA A-a1 receptor in sperm has a stronger relationship with embryo quality at the cleavage stage than the GABA B-R2 receptor.

Keywords: GABA receptors; Intracytoplasmic sperm injection; Oligospermia; Semen analysis; Spermatozoa; Teratozoospermia

Received: August 1, 2020 · Revised: October 15, 2020 · Accepted: October 17, 2020 Corresponding author: Samur Thanoi

Department of Anatomy, Faculty of Medical Science, Naresuan University, Phitsanulok 65000, Thailand

Tel: +66-55-964700 Fax: +66-55-964770 E-mail: samurt@nu.ac.th

*This study was supported by the Thailand Research Fund (TRF) and Naresuan University Research Fund. PK was supported by the Royal Golden Jubilee Ph.D. Program, Thailand.

This is an Open Access article distributed under the terms of the Creative Commons Attribution Non-Commercial License (http://creativecommons.org/licenses/by-nc/4.0/) which permits unrestricted non-commercial use, distribution, and reproduction in any medium, provided the original work is properly cited.

\section{Introduction}

Gamma-aminobutyric acid (GABA) is known to be a major inhibitory neurotransmitter in the central nervous system. Moreover, GABA has also been reported to play functional roles in peripheral organs such as the testis, ovary, uterus, pancreas, and adrenal glands [1]. It plays a role in the regulation of testicular and sperm function through its receptors (especially GABA $A$ and $B$ receptors). Several subunits of GABA $A$ and $B$ receptors have been detected in sperm 
[2-5]. The GABA A receptor alpha 1 (a1) subunit is localized on the head of sperm, as well as the GABA B receptor $\mathrm{R} 2$ subunit $[6,7]$. Although the specific localization of the GABA receptor is not clearly defined, there is evidence that the GABA receptor alpha subunit is localized in the equatorial segment of the human sperm head [8]. Activation of the GABA A receptor, a ligand gated-chloride ion channel receptor, induces hyperpolarization of the cell membrane, whereas the GABA B receptor, a G-protein-coupled receptor, is involved in the opening of calcium and potassium channels [7]. Many studies investigated the role of GABA and its receptors in sperm functions that are important for fertilization. The GABA A and $B$ receptors have been reported to be involved in the modulation of sperm kinetic parameters, including sperm motility [9], the stimulation of sperm capacitation [7,10,11], hyperactivation [9,12-14], and the acrosome reaction [2,7,5-18]; however, their physiological relevance remains elusive. Indeed, both GABA and progesterone can act through GABA receptors to promote those sperm functions by triggering the increase of intracellular calcium and chloride ions, cyclic 3,5' adenosine monophosphate (CAMP), and protein tyrosine phosphorylation in the sperm head [10,17]. Nevertheless, no studies have yet investigated levels of GABA receptors in poor-quality sperm.

Several terms are used to refer to men with poor sperm quality. Oligoasthenoteratozoospermic (OAT) men are defined as men with low levels of three sperm parameters (sperm concentration, motility, and morphology), whereas teratozoospermic (TER) men are defined as those with only low levels of normal sperm morphology. OAT and TER men commonly present to infertility clinics [19]. Intracytoplasmic sperm injection (ICSI) is an assisted reproductive technology for the treatment of infertility, which is defined the inability to conceive after 12 months of regular sexual intercourse without contraception [20]. Currently, up to $50 \%$ (reported range, 20\%-70\%) of the infertile population, corresponding to roughly 50-80 million people worldwide, is affected by male factor infertility, which mostly involves low sperm quality $[21,22]$. The ICSI procedure not only bypasses the processes of natural sperm selection for fertilization that naturally occur within the female reproductive tract such as capacitation, hyperactivation, and the acrosome reaction, but it also omits the process of sperm-oocyte plasma membrane fusion [23]. Nevertheless, failures of fertilization and embryo development from ICSI are also found, especially in men with poor sperm quality [24]. A major cause of those failures is delayed oocyte activation, which occurs after triggering intracellular calcium release and oscillations of sperm-borne oocyte activating factors (SOAFs) [25]. During ICSI, the timing of disintegration of the sperm plasma membrane and acrosome, which occurs within the oocyte, has been reported to influence the likelihood of successful ICSI outcomes because it controls the release of
SOAFs [26-29]. Morozumi et al. [26] showed that removing the sperm plasma membrane and acrosome before ICSI could improve the timing of oocyte activation and the first cleavage division. These findings lead to the hypothesis that the remaining components of the intact sperm plasma membrane and acrosome in the ooplasm following ICSI, including GABA receptors, might have an impact on fertilization and embryo development.

Therefore, the present study aimed to determine the levels of mRNA expression of GABA receptors, including GABA A-a1 and $\mathrm{GABA} B-\mathrm{R} 2$ receptors, in the sperm of men with poor sperm quality (OAT and TER men) compared to normozoospermic (NOR) men. The fertilization rate and embryo quality were compared between groups in patients undergoing ICSI. Moreover, to provide further support for our hypothesis, we also evaluated the correlations of mRNA expression of these receptors with sperm parameters, fertilization rate, and embryo quality.

\section{Methods}

\section{Semen samples}

Semen samples were provided from 113 volunteer patients at the Naresuan Infertility Center, Faculty of Medicine, Naresuan University, Thailand. The experimental protocols were approved by the Institutional Review Board of Naresuan University (IRB No. 0549/60). A signed consent form was obtained from all volunteers. The semen samples were collected by masturbation, following 3-4 days of sexual abstinence, and allowed to liquefy for 30-60 minutes at room temperature. The semen samples were then assessed for liquefaction, appearance, viscosity, pH, volume, and sperm quality (including sperm concentration, motility, and morphology) following the World Health Organization (2010) guidelines [30]. An overview of the sample composition is shown in Figure 1. Ninety-nine semen samples with normal appearance, liquefaction, and $\mathrm{pH}$ values along with a sperm concentration $\geq 5 \times 10^{6} / \mathrm{mL}$ were included in this study, while 14 semen samples with a sperm concentration $<5 \times 10^{6} / \mathrm{mL}$ were excluded. The included samples were divided into three groups: NOR $(n=32)$, OAT $(n=22)$, and TER $(n=45)$. The volunteer patients in the NOR group had normal sperm concentrations ( $\geq 15 \times 10^{6} / \mathrm{mL}$ ), progressive motility (PR; $\geq 32 \%$ ) and morphology (normal form $\geq 4 \%$ ), while those in the OAT group had abnormal sperm concentrations $\left(<15 \times 10^{6} / \mathrm{mL}\right), \mathrm{PR}(<32 \%)$, and morphology (normal form $<4 \%$ ). Volunteer patients with normal sperm concentration and PR, but abnormal sperm morphology, were defined as TER men.

\section{Semen analysis}

A drop $(\sim 10 \mu \mathrm{L})$ of the semen sample was loaded into a Makler counting chamber. Sperm concentration and motility were recorded 


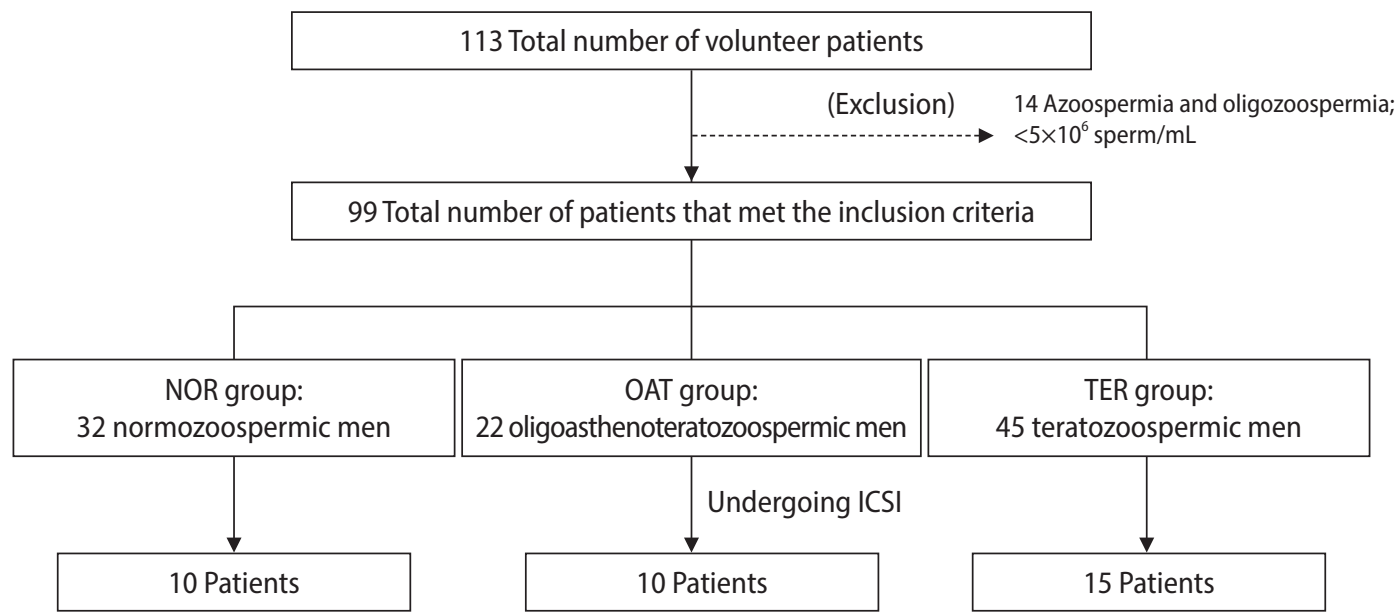

Figure 1. Schematic overview of the sample composition. ICSI, intracytoplasmic sperm injection.

using an Optikam microscope camera and software (OPTIKA Microscopes, Ponteranica, Italy) under a bright-field microscope at $\times 200$ magnification. The average number of sperm per 10 squares was defined as the sperm concentration $\left(\times 10^{6} \mathrm{sperm} / \mathrm{mL}\right)$. The sperm were also graded into PR, non-progressive motility (NP), and immotility. The total of motile sperm was defined as the sum of PR and NP. For sperm morphology analysis, a drop of semen was smeared on a glass slide, allowed to air-dry, fixed, and stained using the Diff-Quick stain. Approximately 200 sperm were examined for sperm morphology under a bright-field microscope at $\times 1,000$ magnification. The percentage of sperm with normal and abnormal morphology was calculated. The label of each slide for the sperm morphology analysis was blinded. For the study of gene expression, all semen samples were stored at $-80^{\circ} \mathrm{C}$ until used.

\section{Reverse transcription-polymerase chain reaction analysis}

Total RNA was isolated from 5-10 $\times 10^{6}$ sperm in each sample using the TRIzol reagent (Invitrogen, Carlsbad, CA, USA) and chloroform. The RNA was converted to complementary DNA (CDNA) using a qScript XLT CDNA Supermix (Quanta Biosciences, Beverly, MA, USA) according to the manufacturer's protocol. The synthesized CDNA was used as a template for reverse transcription-polymerase chain reaction. Oligonucleotide primers for GABA receptors, GABA A- $a 1$ and GABA B-R2 receptors, and GAPDH (reference gene used as an internal control) genes were used following previous studies [31-33], as shown in Table 1. Complementarity with other sequences in the human genome was searched using BLAST on the NCBI website (freely available at https://blast.ncbi.nlm.nih.gov/Blast.cgi). The oligonucleotide primers were synthesized by Macrogen (Macrogen Inc., Seoul, Korea). Each PCR product was amplified from $2 \mathrm{ng}$ of CDNA template using $2 \times$ SensiFAST SYBR No-Rox (BIO-98005, Bioline, London, UK) and performed on the LineGene 9600 Plus QPCR system (Bioer,
Hangzhou, China). The expression levels of GABA receptor genes were normalized to the GAPDH gene and represented as relative mRNA expression values [34]

\section{ICSI procedure, fertilization rate, and embryo quality assessments}

In total, 35 volunteer patients received ICSI treatment at the Naresuan Infertility Centre, Faculty of Medicine, Naresuan University, Thailand, including 10 of the 32 patients in the NOR group, 10 of the 22 patients in the OAT group, and 15 of the 45 patients in the TER group (Figure 1). The female partner of each patient undergoing ICSI underwent controlled ovarian stimulation using a standard protocol (a gonadotropin-releasing hormone antagonist protocol), and oocyte retrieval, as previously described [35]. After incubation for 3 hours, oocyte denudation was performed using a hyaluronidase solution (80 IU/mL hyaluronidase in FertiCult Flushing Medium; FertiPro NV, Beernem, Belgium). Sperm were prepared using two-layer density gradient centrifugation, with $45 \%$ and $90 \%$ of Sil-Select solutions (FertiPro NV), following the standard swim-up method. After ICSI, the fertilization rate and embryo quality were assessed. The fertilization of the injected oocyte was monitored on day 1 ( 16-18 hours after ICSI). An embryo that showed two polar bodies (first and second polar bodies) and two pronuclei was defined as a fertilized oocyte. Embryo quality in each stage of embryo development was then observed from day 2 to 5 after ICSI, including the cleavage stage on day 3; the morula stage on day 4; and the blastocyst stage on day 5 . The cell number, symmetry, and fragmentation were used to verify the embryo quality at the cleavage stage. A good-quality embryo was defined as having 6-8 cells and less than $10 \%$ cytoplasmic fragmentation [36]. At the morula stage, an embryo with evidence of compaction was defined as a good-quality embryo. Blastocyst formation, the inner cell mass (ICM), and the trophectoderm (TE) were 
Table 1. Sequences of oligonucleotide primers for gene expression analysis in human sperm

\begin{tabular}{|c|c|c|c|c|}
\hline Gene & Primer sequence $\left(5^{\prime}-3^{\prime}\right)$ & Annealing temperature $\left({ }^{\circ} \mathrm{C}\right)$ & Product size (bp) & Reference \\
\hline \multirow[t]{2}{*}{ GABAA- $\alpha 1$} & F: AGAAAAACAACACTTACGCTCCA & 57 & 119 & [31] \\
\hline & R: GGGCTTGACCTCTTTAGGTTC & & & \\
\hline \multirow[t]{2}{*}{$G A B A B-R 2$} & F: GGAAGAGGTCACCATGCAG & 66 & 101 & [32] \\
\hline & R: AGTTTCCCAGGTTGAGGATG & & & \\
\hline \multirow[t]{2}{*}{ GAPDH (reference) } & F: GCCTCAAGATCATCAGCAATGCCT & 63 & 104 & [33] \\
\hline & R:TGTGGTCATGAGTCCTTCCACGAT & & & \\
\hline
\end{tabular}

$F$, forward; $R$, reverse.

used to assess embryo quality at the blastocyst stage according to the guideline of Gardner et al. [37]. Blastocyst formation was evaluated using 6 grades based on the degree of expansion and hatching status, as follows: grade 1, early blastocyst; grade 2 , blastocyst; grade 3, full blastocyst; grade 4, expanded blastocyst; grade 5, hatching blastocyst; and grade 6 , hatched blastocyst. The ICM was categorized as grade A, tightly packed with many cells; grade B, loosely grouped with several cells; and grade $C$, very few cells. The TE scores were grade A, many cells forming a cohesive epithelium; grade B, few cells forming a loose epithelium; and grade $C$, very few cells [37]. In this study, the embryo quality at the blastocyst stage was divided into good (3-6 with $A A, A B, B A$, or $B B$ ), moderate (3-6 with $B C, C B$, or $C C$ ), and poor (1-2 regardless of ICM and TE grades and arrested embryos; defective at reaching the blastocyst stage). The fertilization rate and number of embryos of good, moderate, and poor quality in each stage of embryo development were recorded.

To investigate the relationship between the mRNA expression of GABA A- $\alpha 1$ and GABA B-R2 receptors and the embryo quality on cleavage stage, all patients undergoing ICSI were divided into two groups based on the percentage of good-quality embryos at the cleavage stage: good (having a $>50 \%$ proportion of good-quality embryos, $n=22$ ) and poor (having a $\leq 50 \%$ proportion of good-quality embryos, $n=13$ ).

\section{Statistical analysis}

The normality of the data distribution was determined using the Shapiro-Wilk test. The statistical significance of differences between the two groups was analyzed using the Student $t$-test (for parametric data) and the Mann-Whitney test (for nonparametric data). The statistical significance of differences among three groups was analyzed using one-way analysis of variance followed by the Dunnett post hoc test (for parametric data) and the Kruskal-Wallis test followed by the Dunn multiple comparison test (for nonparametric data). Moreover, the Pearson correlation coefficient was used to investigate the relationships of the relative mRNA expression of GABA receptors with sperm parameters, fertilization rate, and embryo quality. The chisquare test was used to compare the categorical variables of groups
Table 2. Comparison of semen parameters in each group of volunteer patients

\begin{tabular}{lccc}
\hline Variable & $\mathrm{NOR}(\mathrm{n}=32)$ & $\mathrm{OAT}(\mathrm{n}=22)$ & $\mathrm{TER}(\mathrm{n}=45)$ \\
\hline Male age $(\mathrm{yr})$ & $36.0 \pm 0.8$ & $37.5 \pm 1.3$ & $37.7 \pm 1.1$ \\
Sperm concentration $\left(\times 10^{6} / \mathrm{mL}\right)$ & $104.5 \pm 14.0$ & $\left.10.2 \pm 0.5^{\mathrm{a}}\right)$ & $91.7 \pm 11.2$ \\
Progressive motility $(\%)$ & $57.8 \pm 2.9$ & $20.6 \pm 1.9^{\mathrm{a})}$ & $52.8 \pm 1.8$ \\
Total motility $(\%)$ & $72.3 \pm 1.9$ & $\left.41.6 \pm 3.4^{\mathrm{a}}\right)$ & $67.2 \pm 1.9$ \\
Normal morphology $(\%)$ & $8.6 \pm 0.4$ & $1.0 \pm 0.2^{\mathrm{a})}$ & $1.2 \pm 0.2^{\mathrm{a})}$ \\
Semen volume $(\mathrm{mL})$ & $3.0 \pm 0.4$ & $2.9 \pm 0.3$ & $2.6 \pm 0.2$ \\
\hline
\end{tabular}

Values are presented as mean \pm standard error of the mean. NOR, normal; OAT, oligoasthenoteratozoospermic; TER, teratozoospermic. ${ }^{\text {a) }}$ Statistically significant compared to NOR group $(p<0.0001)$; Kruskal-Wallis test followed by the Dunn multiple comparison test.

of volunteer patients regarding the rate of fertilization and embryo quality. Statistical significance was considered to be indicated by $p$-values $<0.05$.

\section{Results}

\section{Semen analysis}

The results of the semen analysis for all parameters are shown in Table 2. There were no significant differences in the age of the male patients or semen volume in the OAT and TER groups compared to the NOR group. The OAT group had significantly lower sperm concentration, PR, total motility, and normal morphology than the NOR group. A significantly lower percentage of sperm with normal sperm morphology was found in the TER group than in the NOR group.

\section{The mRNA expression of GABA A- $\alpha 1$ and GABA B-R2 receptors}

Significantly higher mRNA expression of the GABA A-a1 and GABA $B-R 2$ receptors was found in the OAT group than in the NOR group, as shown in Figure 2A (GABA A-a1: $1.7 \pm 0.2$ vs. $1.0 \pm 0.1$ for OAT vs. NOR) and Figure $2 B$ (GABA B-R2: $2.2 \pm 0.3$ vs. $1.0 \pm 0.2$ for OAT vs. NOR); however, the differences between the TER and NOR groups were not significant. 


\section{A}

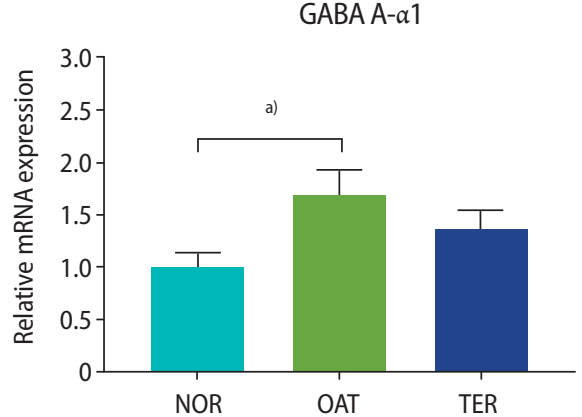

B GABA B-R2

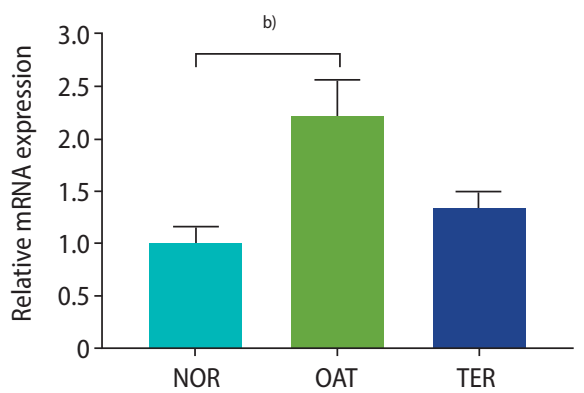

Figure 2. Relative mRNA expression of gamma-aminobutyric acid (GABA) receptors ([A] GABA A-a1, [B] GABA B-R2 receptors) in the oligoasthenoteratozoospermic (OAT) and teratozoospermic (TER) groups compared to normal (NOR) group. Values are presented as mean \pm standard error of the mean. a) $p<0.05,{ }^{\text {b) }} p<0.01$; the KruskalWallis test followed by Dunn multiple comparison.

\section{Fertilization rate and embryo quality after ICSI}

As shown in Table 3, a significantly lower proportion of good-quality embryos at the cleavage stage was found in the OAT and TER groups than in the NOR group. There were no significant differences between groups in the age of the female partner, fertilization rate, or embryo quality in the morula and blastocyst stages.

\section{Correlations of mRNA expression of GABA A- $a 1$ and GABA} $B-R 2$ receptors with sperm parameters, fertilization rate, and embryo quality

High expression of both GABA A-a1 and GABA B-R2 receptors was significantly correlated with low sperm concentration, PR, total motility, and normal morphology (Figure 3). Patients in the good group had significantly lower levels of GABA A-a1 receptor expression than patients in the poor group ( $1.00 \pm 0.16$ vs. $2.24 \pm 0.40)$, but a significant difference was not found for GABA B-R2 receptor expression $(1.32 \pm 0.27$ vs. $1.72 \pm 0.40)$, as shown in Figure 4 . A strong negative correlation was present between the expression of the GABA A-a1 receptor and the percentage of good-quality embryos at the cleavage stage ( $r=-0.464, p=0.005$ ) (Figure 5A); however, a significant correlation was not found for GABA B-R2 receptor expression ( $r=-0.227, p=0.191$ ) (Figure 5B). Moreover, the percentage of good-quality embryos at the cleavage stage exhibited a positive correlation with normal sperm morphology $(r=0.468, p=0.005)$

Table 3. Comparison of baseline characteristics of the female partners of the volunteer patients undergoing ICSI and clinical outcomes after ICSI including fertilization rate and embryo quality

\begin{tabular}{|c|c|c|c|c|c|}
\hline Variable & NOR & OAT & $p$-value & TER & $p$-value \\
\hline Number of patients with ICSI & 10 & 10 & & 15 & \\
\hline Female age (yr) & $34.3 \pm 1.5(29-40)$ & $36.5 \pm 1.3(32-44)$ & $N S^{\mathrm{a})}$ & $36.7 \pm 1.1(30-46)$ & $N S^{\mathrm{a})}$ \\
\hline Number of cycles & 10 & 10 & & 15 & \\
\hline Number of retrieved oocytes & 98 & 66 & & 155 & \\
\hline Number of Mll oocytes injected & 94 & 63 & & 147 & \\
\hline Fertilization rate & $87.2(82 / 94)$ & $82.5(52 / 63)$ & $N S^{b)}$ & $84.4(124 / 147)$ & $N S^{b)}$ \\
\hline Embryo at cleavage stage & & & $0.01^{\mathrm{b})}$ & & $<0.001^{\mathrm{b})}$ \\
\hline GQE & $76.8(63 / 82)$ & $55.8(29 / 52)$ & & $42.9(63 / 124)$ & \\
\hline PQE & $23.2(19 / 82)$ & $44.2(23 / 52)$ & & $57.1(61 / 124)$ & \\
\hline Embryo at morula stage & & & $N S^{b)}$ & & $N S^{b)}$ \\
\hline GQE & $48.8(40 / 82)$ & $46.2(24 / 52)$ & & $40.3(50 / 124)$ & \\
\hline PQE & $51.2(42 / 82)$ & $53.8(28 / 52)$ & & $59.7(74 / 124)$ & \\
\hline Embryo at blastocyst stage & & & $N S^{b)}$ & & $N S^{\mathrm{b})}$ \\
\hline GQE & $22.0(18 / 82)$ & $19.2(10 / 52)$ & & $22.6(28 / 124)$ & \\
\hline MQE & $9.8(8 / 82)$ & $5.8(3 / 52)$ & & $11.3(14 / 124)$ & \\
\hline $\mathrm{PQE}$ & $68.3(56 / 82)$ & $75.0(39 / 52)$ & & $66.1(82 / 124)$ & \\
\hline
\end{tabular}

Values are presented as mean \pm standard error of the mean (range) or percent (number).

ICSI, intracytoplasmic sperm injection; NOR, normal; OAT, oligoasthenoteratozoospermic; TER, teratozoospermic; NS, not significant; MII, metaphase II; GQE, good-quality embryo; $\mathrm{PQE}$, poor-quality embryo; $\mathrm{MQE}$, moderate-quality embryo.

${ }^{\text {a) }}$ One-way analysis of variance followed by the Dunnett post hoc test; ${ }^{\text {b) }}$ Chi-square test. 
(A)

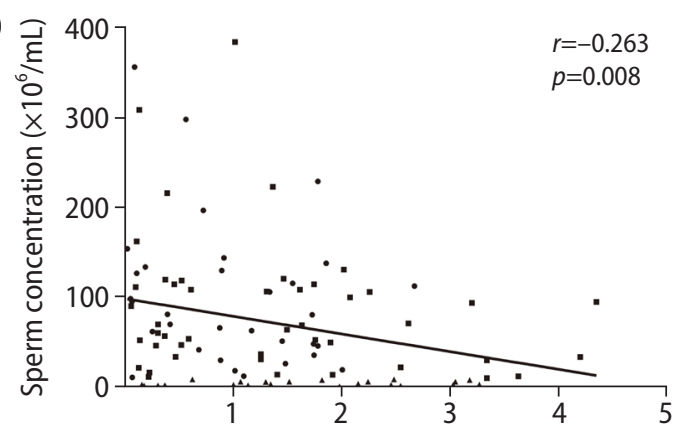

B

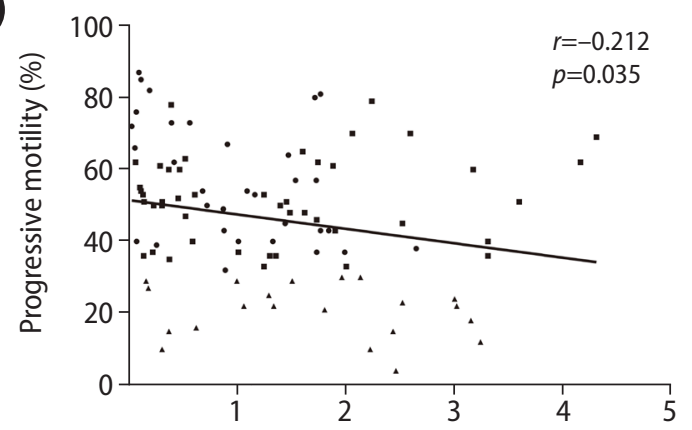

C

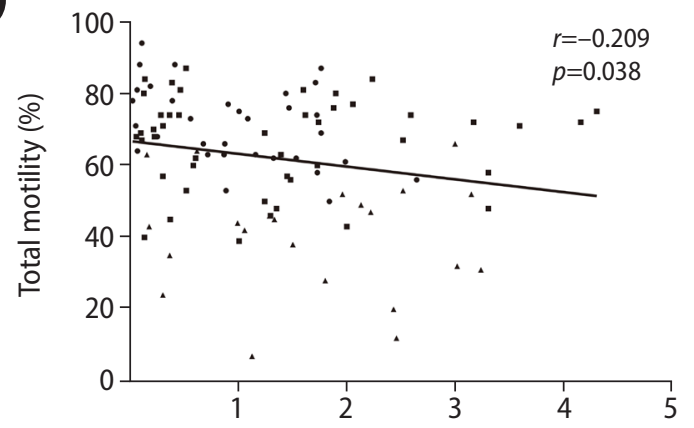

(D)

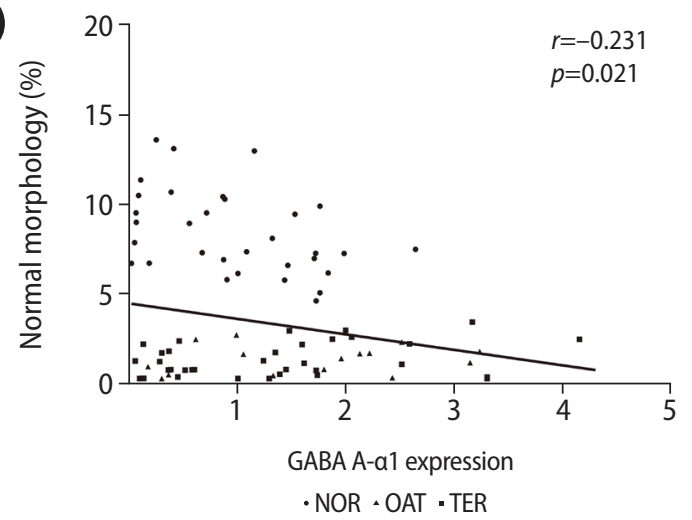

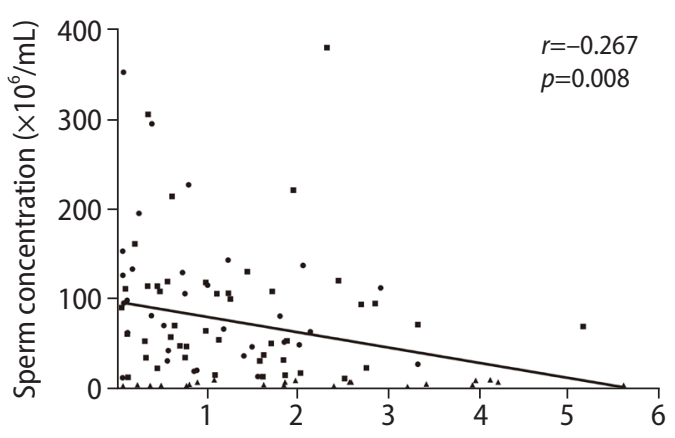
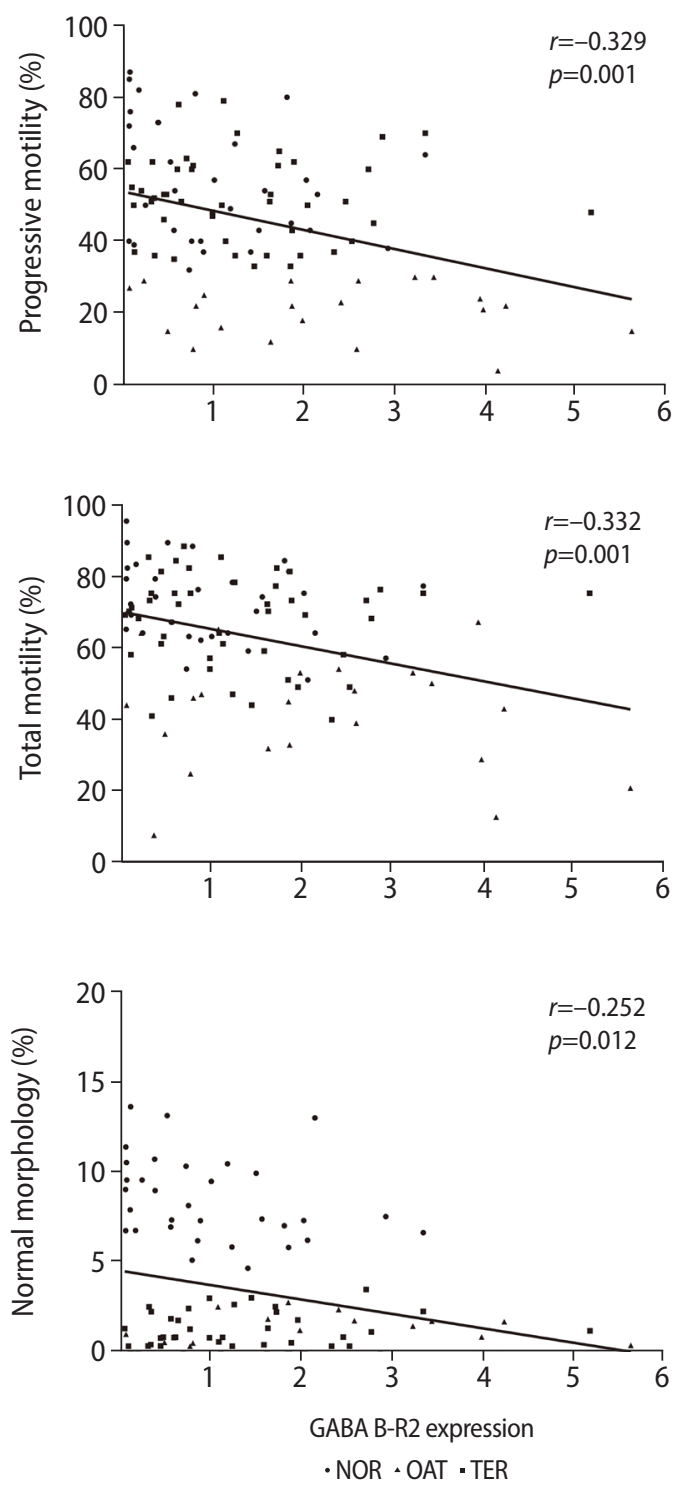

Figure 3. Correlations of mRNA expression of GABA A- $\alpha 1$ (left) and GABA B-R2 (right) receptors with sperm parameters: (A) sperm concentration, (B) progressive motility, (C) total motility, and (D) normal morphology. All data points in the normal (NOR; circles), oligoasthenoteratozoospermic (OAT; triangles) and teratozoospermic (TER; squares) groups are shown. Linear regression line (black line) fitted to all data points. GABA, gamma-aminobutyric acid. 
(Figure 6). There were no significant correlations between the expression of GABA receptors and other outcomes, including fertilization rate and embryo quality at the morula and blastocyst stages (data not shown).

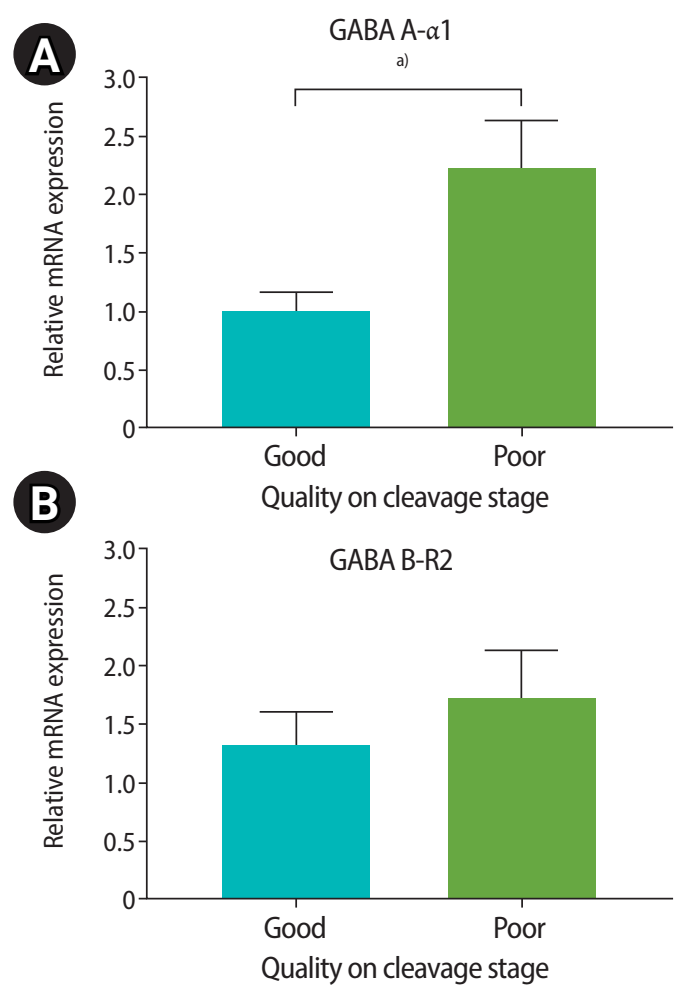

Figure 4. The relative $m R N A$ expression of gamma-aminobutyric acid (GABA) receptors ([A] GABA A- $\alpha 1$, [B] GABA B-R2 receptors) in patients who had the female partner with a good ( $>50 \% \mathrm{GQE})$ and poor ( $\leq 50 \% \mathrm{GQE}$ ) proportion of embryos at the cleavage stage. Values are presented as mean \pm standard error of the mean. GQE, good-quality embryo. ${ }^{\text {a) }} p<0.01$, Student $t$-test.

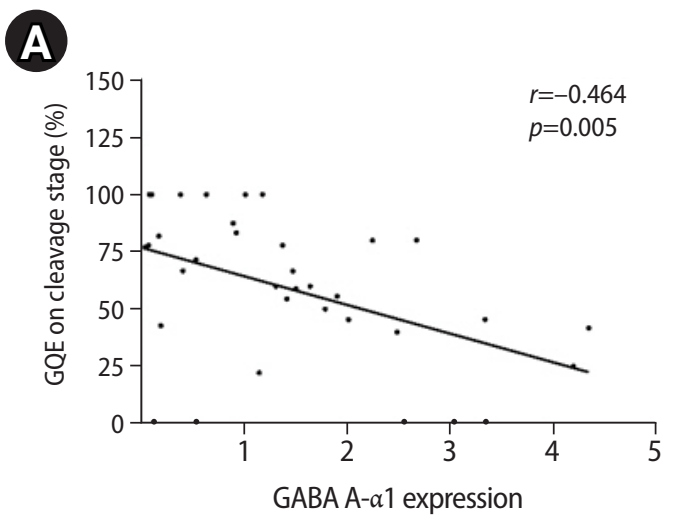

\section{Discussion}

The present study demonstrated high mRNA expression of GABA receptors in the sperm of OAT and TER patients. This result is consistent with our previous study in an animal model of poor sperm quality (in methamphetamine-administered rats), which found significantly higher GABA concentrations as and mRNA expression of the GABA A-a1 receptor and a GABA-synthesizing enzyme in the testis of methamphetamine-administered rats [38]. Additionally, significantly higher GABA A-a1 receptor expression in epididymal sperm was also found in those rats (unpublished data). These results indicate that the expression of GABA receptors changes both in the testis and sperm under conditions of poor sperm quality. GABAergic function in the testis has been reported, including the stimulation of spermatogenesis, Leydig cell proliferation, and testosterone production $[39,40]$. Therefore, it should be noted that the increase of GABA receptors is expected to occur earlier in the testis to compensate for sperm impairment and to maintain the homeostasis of testicular

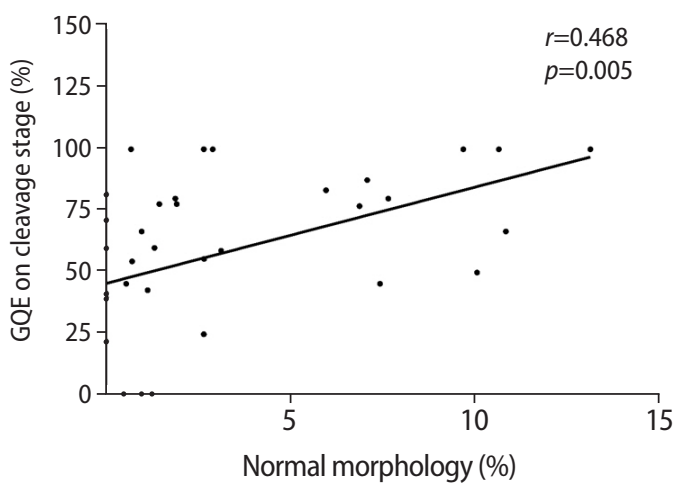

Figure 6. Correlation between the percentage of good-quality embryos (GQEs) at the cleavage stage and normal sperm morphology.

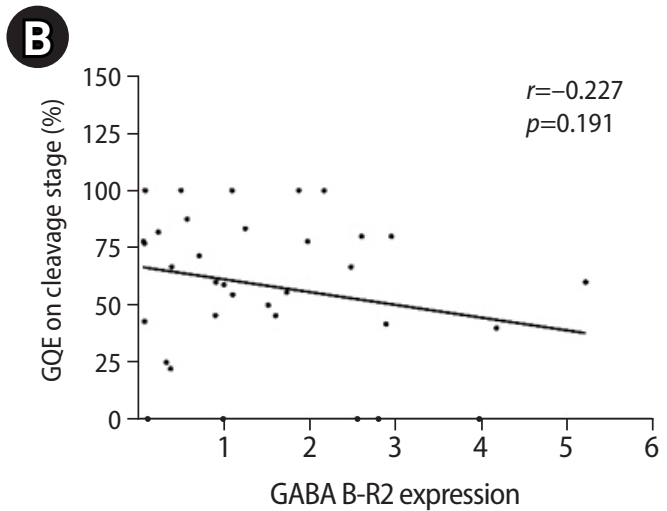

Figure 5. Correlations of mRNA expression of (A) GABA A- $\alpha 1$ and (B) GABA B-R2 receptors with the percentage of good-quality embryos (GQEs) at the cleavage stage. GABA, gamma-aminobutyric acid. 
function; moreover, those receptors remain expressed in epididymal sperm after spermiation. Similarly, changes in GABA receptors were also found in the ejaculated sperm of OAT and TER men. As mentioned above, significantly higher mRNA expression of GABA receptors was found only in OAT men, but not in TER men. Moreover, significant correlations between high levels of GABA receptor expression and low sperm parameters were observed. These results demonstrate that the mRNA levels of GABA receptors depend on the severity of sperm impairment. However, the results of this study only focused on alterations of the transcription process in GABA receptors as measured by mRNA expression, not on the protein expression of GABA receptors in human sperm; the need to clarify the translation process remains a limitation.

Our results for ICSI outcomes are consistent with those of previous studies. Loutradi et al. [41] and Li et al. [42] showed that OAT and TER patients had significantly lower embryo quality at the cleavage stage after ICSI than NOR patients, but no significant difference was found in the fertilization rate. Moreover, Loutradi et al. [41] revealed that there was no significant difference in the embryo quality at the blastocyst stage of OAT patients undergoing ICSI compared to NOR patients. The present study confirmed that the impairment of embryo development in OAT and TER men undergoing ICSI occurs at the cleavage stage. The positive correlation between the percentage of good-quality embryos at the cleavage stage and normal sperm morphology indicates that abnormal sperm morphology might be a major cause of embryo developmental impairment in these patients. There is evidence that OAT and TER men had a high level of sperm DNA fragmentation and chromatin condensation abnormalities [43]. Moreover, several studies have suggested that sperm from those men isolated by density gradient centrifugation still have higher sperm DNA fragmentation and aneuploidy levels than sperm from NOR men, with implications for embryonic cleavage after ICSI [4448]. A review study on the effects of sperm DNA fragmentation on ICSI outcomes found that sperm DNA fragmentation had adverse effects on the pregnancy rate and the timing of blastocyst-stage embryo development, but not on cleavage-stage embryos; however, those relationships were not found in some studies $[49,50]$.

Interestingly, this is the first report on the correlation between GABA receptor expression in sperm and ICSI outcomes. In this study, we showed that significantly higher levels of GABA A-a1 receptor mRNA expression in sperm were found in patients whose female partner had a low percentage of good-quality embryos at the cleavage stage after ICSI. These findings support the hypothesis that the GABA receptors, especially the GABA A-a1 receptor, in human sperm are involved in embryonic cleavage after ICSI. Although sperm immobilization is performed immediately before ICSI to induce sperm plasma membrane disruption, the sperm plasma membrane and ac- rosome remain intact for several hours in the oocyte [51]. As mentioned earlier, during ICSI, the intracellular calcium level in the ooplasm increases in response to triggering of SOAFs from the acrosome after the disintegration of the sperm plasma membrane. Interestingly, it is important for oocyte activation, which has an impact on fertilization and embryo development (from early embryonic development to the blastocyst stage) [23,52]. Taken together, the levels of remaining GABA receptors on the intact sperm head may be associated with delayed oocyte activation because it is known that the activation of GABA receptors results in an increase of intracellular calcium levels in sperm. Using sperm with high levels of GABA receptors in ICSI might disturb the pattern of intracellular calcium oscillations within the ooplasm and cause delays in oocyte activation and cleavage division of the embryo. However, the functional role of GABA receptors in those processes remains unknown.

In summary, the findings of the present study suggest that the mRNA levels of GABA receptors, GABA A-a1 and GABA B-R2 receptors, in sperm can be used as biomarkers to predict male infertility. The finding of high-level mRNA expression of these receptors in sperm reflects poor sperm quality. Moreover, our results demonstrate that the mRNA levels of GABA receptors in human sperm, especially the GABA A-a1 receptor, are associated with embryo quality at the cleavage stage after ICSI.

\section{Conflict of interest}

No potential conflict of interest relevant to this article was reported.

\section{Acknowledgments}

We appreciate the facilities support from Naresuan University and the helpful comments from the Naresuan Infertility Centre. We would like to thank all patients for their participation.

\section{ORCID}

Paweena Kaewman https://orcid.org/0000-0001-7431-4422

Sutisa Nudmamud-Thanoi https://orcid.org/0000-0001-9356-1162

Patcharada Amatyakul https://orcid.org/0000-0003-1523-1584

SamurThanoi

https://orcid.org/0000-0002-6227-7721

\section{Author contributions}

Conceptualization: ST, SNT. Data curation \& Formal analysis: PK, SNT, ST. Methodology: all authors. Project administration: ST, SNT. Visualization: PK, SNT, ST. Writing-original draft: PK. Writing-review \& 
editing: SNT, PA, ST.

\section{References}

1. Gladkevich A, Korf J, Hakobyan VP, Melkonyan KV. The peripheral GABAergic system as a target in endocrine disorders. Auton Neurosci 2006;124:1-8.

2. Hu JH, He XB, Wu Q, Yan YC, Koide SS. Subunit composition and function of GABAA receptors of rat spermatozoa. Neurochem Res 2002;27:195-9.

3. Li SF, Hu JH, Yan YC, Chen YG, Koide SS, Li YP. Identification and characterization of a novel splice variant of beta3 subunit of GAB$A(A)$ receptor in rat testis and spermatozoa. Int J Biochem Cell Biol 2005;37:350-60.

4. Li S, Zhang Y, Liu H, Yan Y, Li Y. Identification and expression of GABAC receptor in rat testis and spermatozoa. Acta Biochim Biophys Sin (Shanghai) 2008;40:761-7.

5. He XB, Hu JH, Wu Q, Yan YC, Koide SS. Identification of GABA(B) receptor in rat testis and sperm. Biochem Biophys Res Commun 2001;283:243-7.

6. He X, Zhang Y, Yan Y, Li Y, Koide SS. Identification of GABABR2 in rat testis and sperm. J Reprod Dev 2003;49:397-402.

7. Kurata S, Hiradate Y, Umezu K, Hara K, Tanemura K. Capacitation of mouse sperm is modulated by gamma-aminobutyric acid (GABA) concentration. J Reprod Dev 2019;65:327-34.

8. Wistrom CA, Meizel S. Evidence suggesting involvement of a unique human sperm steroid receptor/Cl- channel complex in the progesterone-initiated acrosome reaction. Dev Biol 1993; 159:679-90.

9. Calogero AE, Hall J, Fishel S, Green S, Hunter A, D'Agata R. Effects of gamma-aminobutyric acid on human sperm motility and hyperactivation. Mol Hum Reprod 1996;2:733-8.

10. Ritta MN, Bas DE, Tartaglione CM. In vitro effect of gamma-aminobutyric acid on bovine spermatozoa capacitation. Mol Reprod Dev 2004;67:478-86.

11. Jin JY, Chen WY, Zhou CX, Chen ZH, Yu-Ying Y, Ni Y, et al. Activation of GABAA receptor/Cl- channel and capacitation in rat spermatozoa: HCO3- and Cl- are essential. Syst Biol Reprod Med 2009;55: 97-108.

12. Ritta MN, Calamera JC, Bas DE. Occurrence of GABA and GABA receptors in human spermatozoa. Mol Hum Reprod 1998;4: 769-73.

13. Binh NT, Van Thuan N, Miyake M. Effects of liquid preservation of sperm on their ability to activate oocytes and initiate preimplantational development after intracytoplasmic sperm injection in the pig. Theriogenology 2009;71:1440-50.

14. Kon H, Takei GL, Fujinoki M, Shinoda M. Suppression of progester- one-enhanced hyperactivation in hamster spermatozoa by $\mathrm{Y}$-aminobutyric acid. J Reprod Dev 2014;60:202-9.

15. Calogero AE, Burrello N, Ferrara E, Hall J, Fishel S, D'Agata R. Gamma-aminobutyric acid (GABA) A and $B$ receptors mediate the stimulatory effects of GABA on the human sperm acrosome reaction: interaction with progesterone. Fertil Steril 1999;71:9306.

16. Burrello N, Vicari E, D'Amico L, Satta A, D'Agata R, Calogero AE. Human follicular fluid stimulates the sperm acrosome reaction by interacting with the gamma-aminobutyric acid receptors. Fertil Steril 2004;82 Suppl 3:1086-90.

17. Puente MA, Tartaglione CM, Ritta MN. Bull sperm acrosome reaction induced by gamma-aminobutyric acid (GABA) is mediated by GABAergic receptors type A. Anim Reprod Sci 2011; 127:31-7.

18. Gramajo-Buhler MC, Zelarayan L, Lopez Luis A, Sanchez-Toranzo G. Acrosome reaction in the epididymal sperm of Chinchilla lanigera: effect of progesterone, A23187 and stimulation of a GABAA-like receptor. J Exp Zool A Ecol Genet Physiol 2012;317:259_ 65.

19. da Silva MC, Wessler LB, Madeira K, da Silva CC. Male infertility profile in an assisted human reproduction clinic from the south of Santa Catarina, Brazil, from 2012 to 2014. Reprod Climaterio 2017; 32:90-6

20. Vander Borght M, Wyns C. Fertility and infertility: definition and epidemiology. Clin Biochem 2018;62:2-10.

21. Babakhanzadeh E, Nazari M, Ghasemifar S, Khodadadian A. Some of the factors involved in male infertility: a prospective review. Int J Gen Med 2020;13:29-41.

22. Fainberg J, Kashanian JA. Recent advances in understanding and managing male infertility. F1000Res 2019;8:F1000 Faculty Rev670.

23. Neri QV, Lee B, Rosenwaks Z, Machaca K, Palermo GD. Understanding fertilization through intracytoplasmic sperm injection (ICSI). Cell Calcium 2014;55:24-37.

24. Bhilawadikar R, Zaveri K, Mukadam L, Naik S, Kamble K, Modi D, et al. Levels of Tektin 2 and CatSper 2 in normozoospermic and oligoasthenozoospermic men and its association with motility, fertilization rate, embryo quality and pregnancy rate. J Assist Reprod Genet 2013;30:513-23.

25. Anifandis G, Messini Cl, Dafopoulos K, Daponte A, Messinis IE. Sperm contributions to oocyte activation: more that meets the eye. J Assist Reprod Genet 2016;33:313-6.

26. Morozumi K, Shikano T, Miyazaki S, Yanagimachi R. Simultaneous removal of sperm plasma membrane and acrosome before intracytoplasmic sperm injection improves oocyte activation/embryonic development. Proc Natl Acad Sci U S A 2006;103:17661- 
6.

27. Takeuchi T, Colombero LT, Neri QV, Rosenwaks Z, Palermo GD. Does ICSI require acrosomal disruption? An ultrastructural study. Hum Reprod 2004;19:114-7.

28. Seita Y, Ito J, Kashiwazaki N. Removal of acrosomal membrane from sperm head improves development of rat zygotes derived from intracytoplasmic sperm injection. J Reprod Dev 2009;55: 475-9.

29. Katayama M, Sutovsky P, Yang BS, Cantley T, Rieke A, Farwell R, et al. Increased disruption of sperm plasma membrane at sperm immobilization promotes dissociation of perinuclear theca from sperm chromatin after intracytoplasmic sperm injection in pigs. Reproduction 2005;130:907-16.

30. World Health Organization. WHO laboratory manual for the examination and processing of human semen. Geneva: World Health Organization; 2010.

31. Chen ZA, Bao MY, Xu YF, Zha RP, Shi HB, Chen TY, et al. Suppression of human liver cancer cell migration and invasion via the GABAA receptor. Cancer Biol Med 2012;9:90-8.

32. Plummer PN, Colson NJ, Lewohl JM, MacKay RK, Fernandez F, Haupt LM, et al. Significant differences in gene expression of GABA receptors in peripheral blood leukocytes of migraineurs. Gene 2011;490:32-6.

33. Yang L, Yu SJ, Hong Q, Yang Y, Shao ZM. Reduced expression of TET1, TET2, TET3 and TDG mRNAs are associated with poor prognosis of patients with early breast cancer. PLoS One 2015;10: e0133896.

34. Livak KJ, Schmittgen TD. Analysis of relative gene expression data using real-time quantitative PCR and the 2(-Delta Delta C(T)) Method. Methods 2001;25:402-8.

35. SHRE Working Group on Ultrasound in ART; D'Angelo A, Panayotidis C, Amso N, Marci R, Matorras R, et al. Recommendations for good practice in ultrasound: oocyte pick up. Hum Reprod Open 2019;2019:hoz025.

36. Alpha Scientists in Reproductive Medicine and ESHRE Special Interest Group of Embryology. The Istanbul consensus workshop on embryo assessment: proceedings of an expert meeting. Hum Reprod 2011;26:1270-83.

37. Gardner DK, Lane M, Stevens J, Schlenker T, Schoolcraft WB. Blastocyst score affects implantation and pregnancy outcome: towards a single blastocyst transfer. Fertil Steril 2000;73:1155-8.

38. Kaewman P, Nudmamud-Thanoi S, Thanoi S. GABAergic alterations in the rat testis after methamphetamine exposure. Int J Med Sci 2018;15:1349-54.

39. Geigerseder C, Doepner RF, Thalhammer A, Krieger A, Mayerhofer A. Stimulation of TM3 Leydig cell proliferation via GABA(A) receptors: a new role for testicular GABA. Reprod Biol Endocrinol 2004;
$2: 13$.

40. Hu JH, Zhang JF, Ma YH, Jiang J, Yang N, Li XB, et al. Impaired reproduction in transgenic mice overexpressing Gamma-aminobutyric acid transporter I (GAT1). Cell Res 2004;14:54-9.

41. Loutradi KE, Tarlatzis BC, Goulis DG, Zepiridis L, Pagou T, Chatziioannou $E$, et al. The effects of sperm quality on embryo development after intracytoplasmic sperm injection. J Assist Reprod Genet 2006;23:69-74.

42. Li B, Ma Y, Huang J, Xiao X, Li L, Liu C, et al. Probing the effect of human normal sperm morphology rate on cycle outcomes and assisted reproductive methods selection. PLoS One 2014;9: e113392.

43. Sivanarayana T, Krishna ChR, Prakash GJ, Krishna KM, Madan K, Rani BS, et al. CASA derived human sperm abnormalities: correlation with chromatin packing and DNA fragmentation. J Assist Reprod Genet 2012;29:1327-34.

44. Calogero AE, De Palma A, Grazioso C, Barone N, Romeo R, Rappazzo $G$, et al. Aneuploidy rate in spermatozoa of selected men with abnormal semen parameters. Hum Reprod 2001;16:11729.

45. Huang CC, Lin DP, Tsao HM, Cheng TC, Liu CH, Lee MS. Sperm DNA fragmentation negatively correlates with velocity and fertilization rates but might not affect pregnancy rates. Fertil Steril 2005;84: 130-40.

46. Komiya A, Kato T, Kawauchi Y, Watanabe A, Fuse H. Clinical factors associated with sperm DNA fragmentation in male patients with infertility. ScientificWorldJournal 2014;2014:868303.

47. Sivanarayana T, Ravi Krishna C, Jaya Prakash G, Krishna KM, Madan K, Sudhakar G, et al. Sperm DNA fragmentation assay by sperm chromatin dispersion (SCD): correlation between DNA fragmentation and outcome of intracytoplasmic sperm injection. Reprod Med Biol 2013;13:87-94.

48. Borges E Jr, Zanetti BF, Setti AS, Braga DP, Provenza RR, laconelli A Jr. Sperm DNA fragmentation is correlated with poor embryo development, lower implantation rate, and higher miscarriage rate in reproductive cycles of non-male factor infertility. Fertil Steril 2019;112:483-90.

49. Kim GY. What should be done for men with sperm DNA fragmentation? Clin Exp Reprod Med 2018;45:101-9.

50. Chi HJ, Kim SG, Kim YY, Park JY, Yoo CS, Park IH, et al. ICSI significantly improved the pregnancy rate of patients with a high sperm DNA fragmentation index. Clin Exp Reprod Med 2017;44: 132-40.

51. Yanagimachi R. Intracytoplasmic injection of spermatozoa and spermatogenic cells: its biology and applications in humans and animals. Reprod Biomed Online 2005;10:247-88.

52. Kurokawa M, Fissore RA. ICSI-generated mouse zygotes exhibit al- 
tered calcium oscillations, inositol 1,4,5-trisphosphate receptor-1 down-regulation, and embryo development. Mol Hum Reprod 2003;9:523-33. 